\title{
Power Envelope Variation Mitigation of LTE System using Constellation-Shaping Approach
}

\author{
Joolan Nazar Alwan \\ University of \\ Information Technology \\ and Communication, \\ Baghdad, Iraq
}

\author{
Zahraa Ali Jawad \\ Department of \\ Electronics and Communication, \\ College of Engineering \\ University of Baghdad, \\ Baghdad, Iraq
}

\begin{abstract}
Peak to average power ratio minimization techniques in orthogonal-frequency-division-multiplexing (OFDM) are hot researching topics since more than two decades. In this work, a novel approach without side information has been proposed, which is based on the constellation re-mapping to randomize the order of data as much as possible, such that the correlation between data vanished. The suggested method will exchange the constellation points with other constellation points, which leads to reformatting the constellation mapping, where by which the new OFDM symbol will be created with lower power envelope variation. In contrast with other methods which addressed in the literature, there will be no restrictions on the mapping family or even the alphabet order. Furthermore, the performance of the bit error rate (BER) has not been affected. Moreover, the problem of the computational complexity has not been affected, where the number of mathematical operations has an ignorable effect.
\end{abstract}

\section{General Terms}

Digital Communication, Orthogonal Frequency Division Multiplexing, Peak to Average Power Ratio.

\section{Keywords}

OFDM, Power envelope variation, Constellation mapping, BER performance.

\section{INTRODUCTION}

Technology is growing up and the telecommunication systems have an important part of this development. Since the first generation of mobile systems in 1980s, the development of digital signal processors (DSP) was growing as well. That is, the discrete Fourier transform (DFT) and inverse DFT (IDFT) algorithms have been simply implemented as fast versions, inverse fast Fourier transform (IFFT) and fast Fourier transform (FFT), using the DSP processors. Therefore, the implementation of the orthogonal frequency division multiplexing (OFDM) becomes as simple as possible. However, the OFDM can be considered as one type of the multicarrier modulation systems. OFDM system allows overlapping of subcarriers orthogonally, therefore, the spectrum efficiency has enhanced dramatically, then, OFDM system could support higher data rates than frequency division multiplexing (FDM) or the single carrier traditional modulation methods. Moreover, OFDM is free inter-symbolinterference (IS), because of the cyclic prefix period, which is a copy of the last part of the OFDM symbol attached to the beginning of the OFDM symbol, that is the inter-carrierinterference (ICI) will be vanished as well, furthermore, OFDM supports the capability to tackle the channels consist of more than one direct path, due to reflections and scattering, which is called multipath and frequency selective channels [1]. OFDM has been employed in different modern communication systems, such as long term evolution (LTE) [2], Worldwide Interoperability for Microwave Access (WiMAX) [3], Because of multicarrier constructive addition, the power envelope variation will be out of band leading to inter-modulation distortion. That is, multicarrier systems have the power envelope variation problem, which is traditionally called peak to average power ratio (PAPR) problem. However, OFDM is an important part of the LTE system, especially in the downlink direction. Therefore, the problem of high PAPR has to be resolved.

The power envelope variation mitigation problem can be resolved in different ways, the literature have rich approaches to solve this problem, but there is a trade-off between the bit error rate (BER), the computational complexity, and the PAPR reduction gain. Such schemes are; the amplitude clipping [4], which is degrades the BER performance, tone reservation $[5,6]$, tone injection $[5,6]$, active constellation extension [7], where these methods are blind that do not need to send side information to the receiver to recover the original date, while the not blind are that methods which need to send side information to the receiver to recover the transmitted data, such methods are selected mapping (SLM) $[8,9]$, partial transmit sequences $[9,10]$. To the best of our knowledge, amplitude clipping was the first approach utilized to reduce the high peaks in multicarrier modulation systems, it is the simplest method among the aforementioned algorithms, but amplitude clipping causes out of band radiation and in-band distortion. On the other hand, tone reservation, tone injection and active constellation distortion algorithms need more power for transmission. While the selected mapping and partial transmit sequences are frequency domain methods with higher computational complexity and produce no distortion to the transmitted data but require side information, which reduces the bit rate of the system.

Another location of data manipulation is the constellation stage. In other words, re-shaping the constellation points in such a way to reduce the high power peaks. These methods called constellation shaping algorithms. However, constellation shaping approaches have a limitation, which is applicable to the square-shapes of constellation, that is, constellation re-shaping methods employed with multi-level quadrature amplitude modulation (M-QAM) mapping family $[11,12]$. Furthermore, tone reservation requires more power for transmission, in [13] Dalakas suggested to mitigate the power envelope variation problem, where by which the problem enhanced without dependency on the constellation family type, where the constellation re-shaping has been done in the time domain, but there will be BER performance degradation. In this paper, a method which is blind, no need for the side information will be introduced. The suggested method did not depend on the constellation family type, works 
in the frequency domain, without degradation in the BER performance. Our proposed method adds every fourconsecutive points with the next four-consecutive points and subtract them. Thus, the points order will be changed; thereby the PAPR will be reduced significantly without affecting the BER performance for any M-ary constellation family and any size of OFDM symbol, N. The rest of this paper organized as follows; section 2 discusses the OFDM system and the PAPR problem, section 3 depicts the suggested algorithm, section 4 shows the results and discusses them, last but not least, section 5 concludes our work.

\section{OFDM AND PAPR MATHEMATICAL MODELS}

The general structure of the OFDM system is illustrated in Figure 1. Binary data is mapped on an M-QAM or M-PSK constellation diagram demultiplexing the serial stream to produce the parallel samples, using the serial-to-parallel functionality. Now the signal will be amplitude modulated using the IDFT-function. Converting back to the serial form will be achieved using the parallel-to-serial multiplexer. The cyclic prefix will be attached, then, the signal will be sent to the receiver. The reverse of the above operations will be accomplished at the receiver. These operations described above can be formulated as,

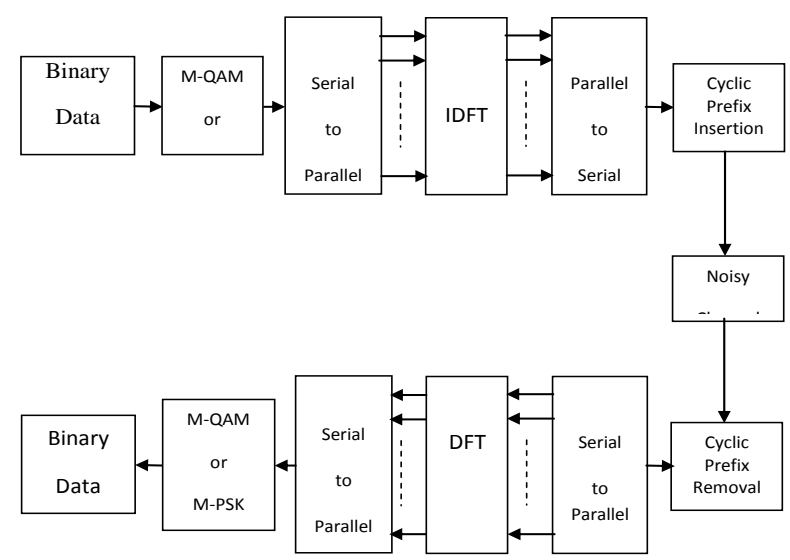

Fig 1: Basic OFDM block diagram that utilizing IDFT/DFT transformation pair

$x(n)=\frac{1}{\sqrt{N}} \sum_{k=0}^{N-1} X(k) \cdot e^{j \frac{2 \pi n k}{N}}, \quad 0 \leq n, k \leq N-1$

where $n$ represents the discrete-time index and $k$ is the frequency-domain index. The last expression stands for the IDFT of the input sequence $X(k)$. That is, Eq. 1 shows an addition of subcarriers, if there are some subcarriers in-phase, the subcarriers will be added constructively, thus, high peaks will arise, causing the PAPR, that is, the PAPR can be defined as,

$$
P t A=\frac{\max |x(n)|^{2}}{E\left[|x(n)|^{2}\right]}
$$

where $E[\cdot]$ is the expectation operation which gives the average value. To capture these peaks in PtA, the complementary cumulative distribution function $(C C D F)$ may used for this job as,

$$
C C D F(P t A)=\operatorname{Pr}(P t A>\eta)
$$

\section{SUGGESTED ALGORITHM}

In this work, we proposed to reduce the PAPR by re-shaping the constellation points. The reshaping procedures are: adding each two constellation points, separated by $N / 2$ samples, to generate the first new constellation point, then subtracting them to generate the other point. For example, $\mathbf{X}=\left[\begin{array}{llllllll}X_{1} & X_{2} & X_{3} & X_{4} & X_{5} & X_{6} & X_{7} & X_{8}\end{array}\right]^{\mathrm{T}}$, Where $N=8$, then, the new

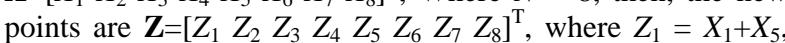
$Z_{5}=X_{1}-X_{5}, \quad Z_{2}=X_{2}+X_{6}, \quad Z_{6}=X_{2}-X_{6}, \quad Z_{3}=X_{3}+X_{7}, \quad Z_{7}=X_{3}-X_{7}$, $Z_{4}=X_{4}+X_{8}$, and $Z_{8}=X_{4}-X_{8}$. Unlike [14], where Taher added/subtracted every two consecutive points, while our suggested method reduces the correlation between constellation points more than Taher's method [14]. That is, the suggested method can be formulated as

$Z=\left[\begin{array}{c}Z_{n} \\ Z_{\frac{N}{2}+1}\end{array}\right]=\left[\begin{array}{cc}1 & 1 \\ 1 & -1\end{array}\right]\left[\begin{array}{c}X_{n} \\ X_{\frac{N}{2}+1}\end{array}\right]$

After the constellation re-shaping in the last expression, the signal $\mathbf{Z}$ can be fed to the conventional IDFT block, as in Figure 1,

$x(n)=\frac{1}{\sqrt{N}} \sum_{k=0}^{N-1} Z(k) \cdot e^{j \frac{2 \pi n k}{N}}, \quad 0 \leq n, k \leq N-1$

where $Z(k)$ computed from Eq. 4, which represent the new constellation points. That is, our algorithm eliminates the requirement for the additional multiplications operations of [15], which degrades the BER performance as well as increases the computational complexity. Thus, using our suggested method, the computational complexity has been diminished significantly. Moreover, the proposed approach does not need to transmit any side information to recover the original data, because of the suggested algorithm can be reversed at the receiving end to be,

$Z=\left[\begin{array}{c}X_{n}^{r} \\ X_{\frac{N}{2}+1}^{r}\end{array}\right]=\frac{1}{2}\left[\begin{array}{cc}1 & 1 \\ 1 & -1\end{array}\right]\left[\begin{array}{c}Z_{n}^{r} \\ Z_{\frac{N}{2}+1}^{r}\end{array}\right]+W$

In the last expression, $Z_{n}^{r}$ and $Z_{\frac{N}{2}+1}^{r}$ stand for $n^{\text {th }}$ and

$(\mathrm{N} / 2+1)^{\text {th }}$ samples at the receiver end, while $W$ is the additive white Gaussian noise. One drawback in our suggested method is that it requires $3 \mathrm{~dB}$ more power to decode the original data as stated in Eq. 6, where the factor $1 / 2$ is presented. It is worth mention that the BER performance will not be affected, since the minimum distance between the new constellation points can be derived as follows: starting with the phases of the $M$ PSK family, they are defined as [16],

$\theta_{m}=\frac{2 \pi(m-1)}{M}, m=1,2 \cdots M$

thus, $M$-PSK signal is,

$$
\begin{aligned}
Y_{m}(t) & =\sqrt{\frac{2 E}{T}} \cos \theta_{m} \cos \left(2 \pi f_{c} t\right)-\sqrt{\frac{2 E}{T}} \sin \theta_{m} \sin \left(2 \pi f_{c} t\right) \\
& =Y_{m 1} \lambda_{1}(t)+Y_{m 2} \lambda_{2}(t), \quad 0 \leq t<T
\end{aligned}
$$

where $\lambda_{1}(t)$ and $\lambda_{2}(t)$ are the basis functions, $T$ is the period of the basis function, $f_{c}$ is the carrier frequency, and $E$ is the symbol energy. Hence, the constellation point set could be,

Where $\eta$ is the clipping level (threshold). 
$Y_{m}=\left[\sqrt{E} \cos \left(\frac{2 \pi(m-1)}{M}\right), \sqrt{E} \sin \left(\frac{2 \pi(m-1)}{M}\right)\right]$

assuming the two points are $Y_{\mathrm{m}}$ and $Y_{\mathrm{n}}$, then, the summation of $Y_{\mathrm{m}}$ with $Y_{\mathrm{n}}$ will be $Z_{1}$ as,

$Z_{1}=\left[\begin{array}{l}\sqrt{E}\left(\cos \left(\frac{2 \pi(m-1)}{M}\right)+\cos \left(\frac{2 \pi(n-1)}{M}\right)\right), \\ \sqrt{E}\left(\sin \left(\frac{2 \pi(m-1)}{M}\right)+\sin \left(\frac{2 \pi(n-1)}{M}\right)\right)\end{array}\right]$

where $m, n=1,2 \ldots M$, the subtraction of $Y_{m}$ and $Y_{n}$ is $Z_{2}$,

$Z_{2}=\left[\begin{array}{l}\sqrt{E}\left(\cos \left(\frac{2 \pi(m-1)}{M}\right)-\cos \left(\frac{2 \pi(n-1)}{M}\right)\right), \\ \sqrt{E}\left(\sin \left(\frac{2 \pi(m-1)}{M}\right)-\sin \left(\frac{2 \pi(n-1)}{M}\right)\right)\end{array}\right]$

Thus, the Euclidian distance between $Z_{1}$ and $Z_{2}$ is,

$d_{Z_{1} Z_{2}}^{(M-P S K)}=\left\|Z_{2}-Z_{1}\right\|=2 \sqrt{E}$

However, the minimum distance of the $M$-PSK signal is [17],

$$
d_{\min }^{(M-P S K)}=2 \sqrt{E} \sin \frac{\pi}{M}
$$

by comparing (12) with (13), a direct conclusion can be deduced which is the distance $d_{Z_{1} Z_{2}}^{(M-P S K)}$ will never by less than the distance $d_{\min }^{(M-P S K)}$, in other words,

$d_{\min }^{(M-P S K)} \geq d_{\min }^{(M-P S K)}$

Following the same procedure, the corresponding expression for the $M$-QAM diagram will be,

$d_{\min }^{(M-Q A M)} \geq d_{\min }^{(M-Q A M)}$

In spite of everything, about $3 \mathrm{~dB}$ more power requirement, the proposed algorithm gives another advantages, such as the dramatically reduced computational complexity. However, according to the theory the computations that are needed are only the summation operations, thus, the number of addition operations is only $2 N$ operations,

$A_{R V-C R}=2 N$

That is, Eq. 16 shows that there is no multiplication operations in our proposed method.

\section{RESULTS AND DISCUSSION}

This section introduces the results and the discussion of the proposed algorithm. The simulation will import some of the LTE OFDM symbol sizes with the most used baseband modulation orders. Thus, the OFDM signals will be produced according to the LTE symbols sizes such as $N=256$, and $N=$ 1024 , both sizes will be conducted with $M=4,16$ and 64 levels. Table 1 shows these parameters settings.
Table 1:Setting the three scenarios for the suggested method

\begin{tabular}{|c|c|c|}
\hline Scenario & $M$ & $N$ \\
\hline 1 & 4 & 256,1024 \\
\hline 2 & 16 & 256,1024 \\
\hline 3 & 64 & 256,1024 \\
\hline
\end{tabular}

Figure 2 shows the PAPR comparison between OFDM signals of size $N=256$ with 4-QAM and 4-PSK, this size corresponds to 3GPP-LTE. However, the reduced PAPR amount is, approximately, $3 \mathrm{~dB}$. In other words, the PAPR reduced from $12.5 \mathrm{~dB}$ to $9.5 \mathrm{~dB}$, for both baseband modulation families. Hence, the re-ordering of the constellation points is the reason behind the PAPR reduction gain. Figures 3 and 4 explain the same OFDM size, $N=256$, but when $M=16$ and 64 levels, respectively. It is shown that the PAPR was reduced by $3 \mathrm{~dB}$ for both families, 16-PSK and 16-QAM families. On the other hand, the extra computations are only due the additional additions operations, which is only $2 N$ regardless the constellation order, thus there are 512 addition operations, added to the conventional number of additions in the original IFFT block.

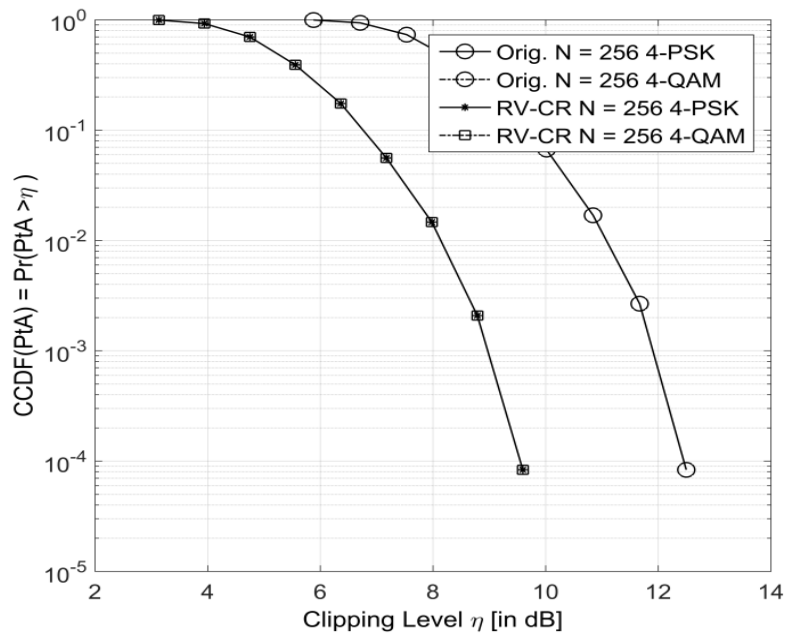

Fig 2: PAPR of original signal and proposed method, $N=$ 256, $M=4$

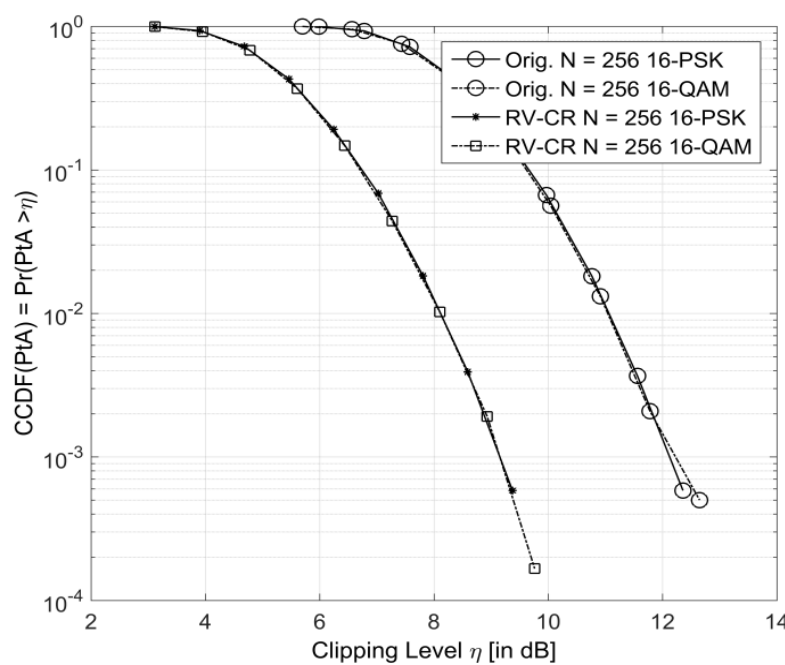

Fig 3: PAPR of original signal and proposed method, $N=$ 256, $M=16$ 


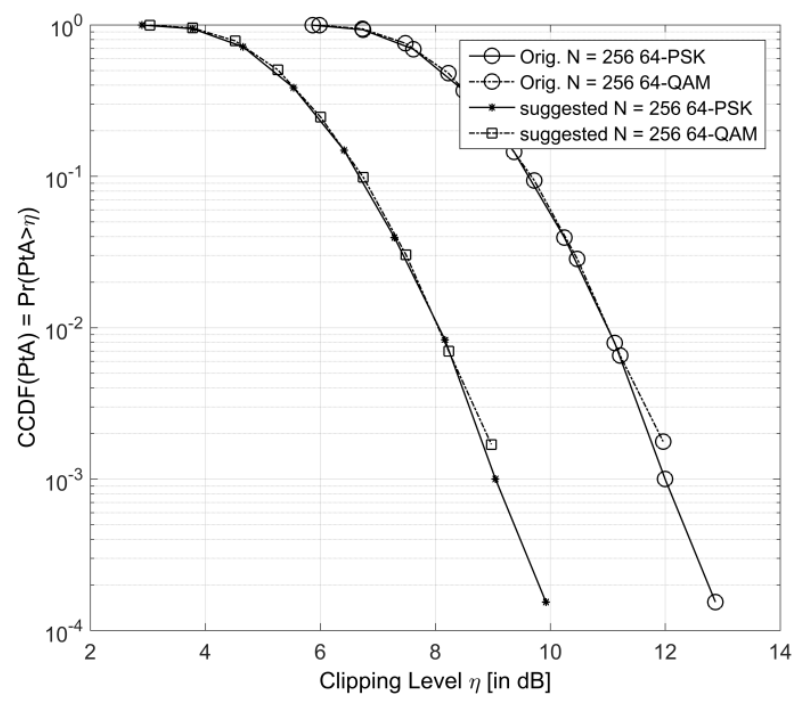

Fig 4: PAPR of original signal and proposed method, $N=$ 256, $M=64$

The same behaviour can be seen for the next scenario, where $N=1024$. That is, for the OFDM signal of size 1024 subcarriers, the conducted constellation orders are $M=4,16$, and 64 levels, for both mapping families, $M$-QAM and $M$ PSK, as given in Table 1. It can be seen in Figure 5, that the PAPR reduction was $3 \mathrm{~dB}$ for both families.

Thus, our suggested method did not has the limitation of the constellation family type, the PAPR was reduced from 14.3 $\mathrm{dB}$ down to $11.3 \mathrm{~dB}$. Figure 6 depicts the PAPR reduction performance for 16-PSK/QAM families, where the OFDM size was 1024 as well. It is shown that the PAPR was reduced from $14.3 \mathrm{~dB}$ to $11.3 \mathrm{~dB}$, for both constellation families.

Figure 7 explains the same fashion, where by using the suggested algorithm, the PAPR can be enhanced by $3 \mathrm{~dB}$, where the PAPR was reduced from $14.2 \mathrm{~dB}$ to $11.2 \mathrm{~dB}$, for the two constellation families, 64-PSK/QAM constellation families. On the other hand, the computational complexity did not increased extremely, there was $2 N$ extra additions operations, in other words, there are 2048 operations of addition beside the original IFFT block.

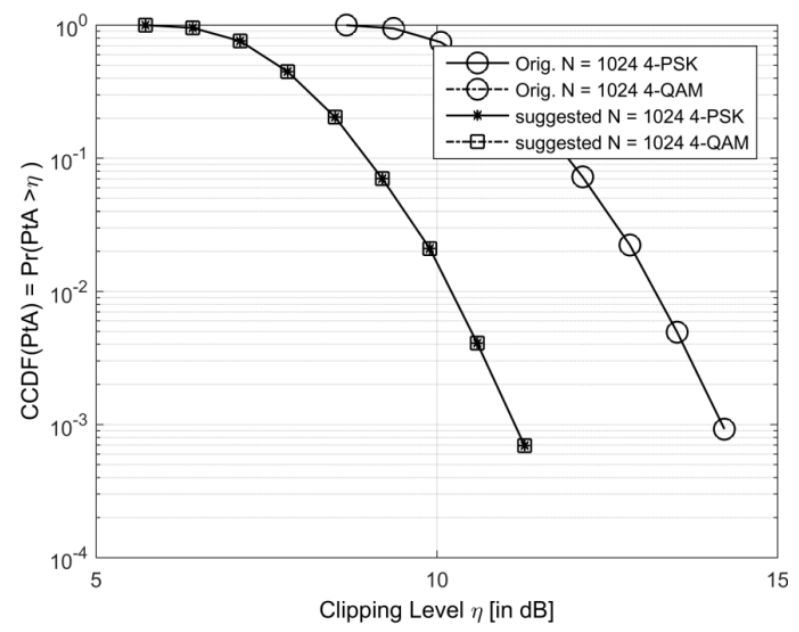

Fig 5: PAPR of original signal and proposed method, $N=$ 1024, $M=4$

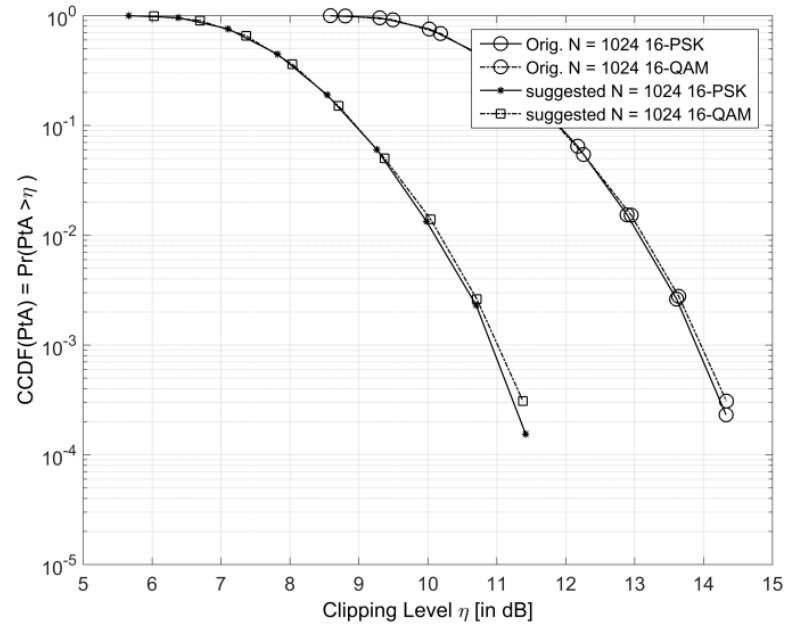

Fig 6: PAPR of original signal and proposed method, $N=$ 1024, $M=16$

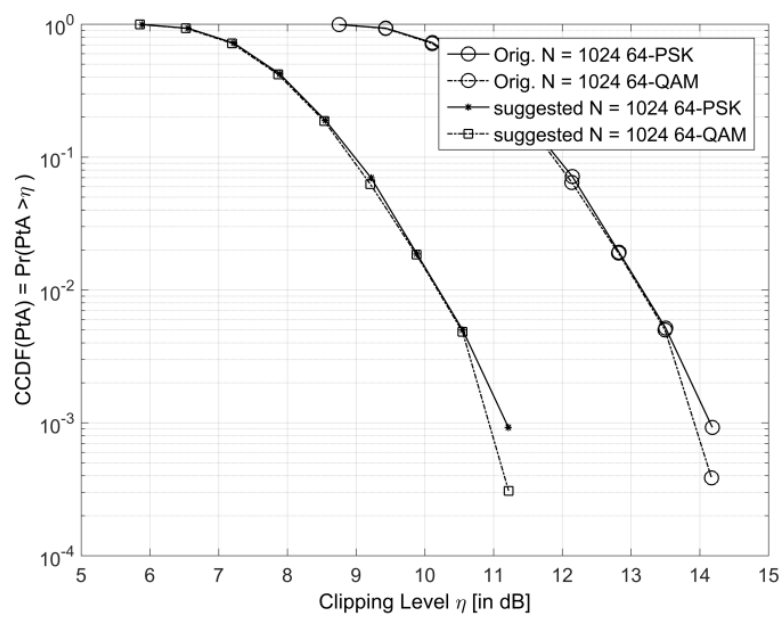

Fig 7: PAPR of original signal and proposed method, $N=$ $1024, M=64$

\section{CONCLUSIONS}

In this paper, a novel fashion to reduce the PAPR for LTE applications has been introduced. Unlike [15] the BER performance did not affect with additional advantages, which is reduced complexity dramatically. Our The designed criteria changes or reshapes the constellation diagram, to randomize the input data in the frequency domain. In other words, eliminates the correlation between the constellation points, that is, the PAPR reduced significantly. Unlike [14], where the constellation reshaping has been achieved in the time domain, leading to BER performance degradation. The power envelope has been enhanced by about $3 \mathrm{~dB}$ more than achieved by [14], where the PAPR was reduced by $1 \mathrm{~dB}$ only. The proposed algorithm can be recommended for the LTE applications, where there is no restrictions to the constellation family, that is why, our future work is to make use of the proposed method for the next generation of communication systems, which is the fifth generation. 


\section{REFERENCES}

[1] Tao, J. and W. Yiyan, "An Overview: Peak-to-Average Power Ratio Reduction Techniques for OFDM Signals", IEEE Transactions on Broadcasting, 2008. 54(2): p. 257268.

[2] Bhat, P., et al., "LTE-advanced: an operator perspective", IEEE Communications Magazine, 2012. 50(2): p. 104114

[3] Ahmadi, S., "An overview of next-generation mobile WiMAX technology", IEEE Communications Magazine, 2009. 47(6): p. 84-98.

[4] Mestdagh, D.J.G., P.M.P. Spruyt, and B. Biran, "Effect of amplitude clipping in DMT-ADSL transceivers", Electronics Letters, 1993. 29(15): p. 1354-1355.

[5] Jung-Chieh, C., et al., "A Suboptimal Tone Reservation Algorithm Based on Cross-Entropy Method for PAPR Reduction in OFDM Systems", IEEE Transactions on Broadcasting, 2011. 57(3): p. 752-756.

[6] Tellado, J., 2000. Multicarrier Modulation With Low PAR: Applications to DSL and Wireless. Kluwer Academic Publishers.

[7] Krongold, B.S. and D.L. Jones, "PAR reduction in OFDM via active constellation extension. IEEE Transactions on Broadcasting, 2003. 49(3): p. 258-268.

[8] Bauml, R.W., R.F.H. Fischer, and J.B. Huber, "Reducing the peak-to-average power ratio of multicarrier modulation by selected mapping", Electronics Letters, 1996. 32(22): p. 2056-2057

[9] Chakrapani, A. and V. Palanisamy, "A survey on CF method, PTS approach, companding technique and time domain methods for PAPR reduction in OFDM systems", European Journal of Scientific Research, 2012. 75(4): p. 624-642.

[10] Muller, S.H. and J.B. Huber, "OFDM with reduced peakto-average power ratio by optimum combination of partial transmit sequences", Electronics Letters, 1997. 33(5): p. 368-369.

[11] Yang, Z. and J. Tao, "A Novel Multi-Points Square Mapping Combined With PTS to Reduce PAPR of OFDM Signals Without Side Information", IEEE Transactions on Broadcasting, 2009. 55(4): p. 831-835.

[12] Cai, L., et al., "A Novel Constellation Reshaping Method for PAPR Reduction of OFDM Signals", IEEE Transactions on Signal Processing, 2011. 59(6): p. 2710 2719.

[13] Park, S.-E., et al., "Tone Reservation method for PAPR Reduction scheme", IEEE 802.16e-03/60, 2003.

[14] Taher, M., et al., "Reducing the PAPR of OFDM Systems by Random Variable Transformation", ETRI Journal, 2013. 35(4): p. 714-717.

[15] Dalakas, V., A.A. Rontogiannis, and P.T. Mathiopoulos, "Time domain constellation shaping technique for peakto-average power ratio reduction", IET Communications, 2009. 3(7): p. 1144-1152.

[16] Proakis, J.G. and M. Salehi, 2008. Digital Communications, 5th Edition. McGraw-Hill Science/Engineering/Math. 1150

[17] Sardar, S., A.H.M.T. Ahmed, and R. Achar. 2015. A new approach using RCMN algorithm for PAPR optimization in LTE SC-FDMA. In 2015 International Conference on Circuit, Power and Computing Technologies (ICCPCT), 2015. 